\title{
A simulation study on the health concerns derived from GSM base station placement
}

\author{
J. P. Muñoz-Gea, J. Vales-Alonso*, J. García-Haro, F. J. González-Castaño \\ Department of Information Technologies and Communications, \\ Polytechnic University of Cartagena, Spain. \\ * Corresponding author. Address: Campus Muralla del Mar, 30202, Cartagena, Spain. \\ phone: +34 968326 588, fax: +34 968325 973, e-mail: javier.vales@upct.es
}

\begin{abstract}
In the last years a great concern has raised among population due to the placement of the base stations (BS) of cellular systems in urban areas. In some cases, it has been addressed with the (re)installation of BSs in far suburban areas. However, in these cases, mobile stations (MS) must raise transmission power. Thus, users may -potentially- suffer a higher radio exposure. In this paper we quantified the variation of the transmission power in this application scenario. The study has been done by means of the OPNET/GSM framework simulation tool. Suitable propagation models and the GSM power control algorithm have been added to the model, in order to compute the MS transmission power in a real environment. Results reveal a significative increment of the MS transmission power when the BS is relocated to suburban areas. Therefore, we conclude that it is necessary to introduce modifications in the GSM network architecture to solve this issue together with the BS placement problem.
\end{abstract}

Index Terms-EMF, Base stations, GSM, Health issues

\section{INTRODUCTION}

One of the main public concerns about wireless cellular telephony systems has been the effect of electromagnetic radiations on human health. This imposes a deployment problem. Traditionally, base stations (BS) were placed in urban areas. Residents nearby complain about the high level of electromagnetic fields (EMF) in their sorroundings, and request an answer to the authorities in order to forbid such antenna placements or to take restrictive actions. In some cases, the solution adopted to reduce electromagnetic fields is to locate the BS in suburban areas. However, in this cases, urban mobile stations (MS) must raise transmission power, and, thus, although their users may not be aware, they may potentially suffer a higher radio exposure.

In this work, we aim to quantify this increment, and decide if it may be significative to raise health concerns. We address this calculation by means of simulation. Through the simulation tool we measure the power transmitted by MSs in both cases: when the BS is located in urban and suburban areas.

The rest of the paper is organized as follows. Section II discusses the effects of EM radiations. Section III presents the OPNET Modeler, the tool used in the simulations. Section IV explains the propagation models used in the different scenarios. Section V presents the GSM power control algorithm. Section VI describes the simulated scenarios, and, finally, Section VII concludes.

\section{DISCUSSION}

International bodies have intensely discussed if EM radiations have effects on human health [1], [2]. They have seeked an answer to whether BSs transmitter/antennas, MS sets, and other types of portable transceivers convey potential risk to human health. Altough definitive conclusions have not been reported, there is a general agreement on the need of prevention, i.e. EMF exposure should be minimal to avoid possible risks.

The International Commission on Non-Ionizing Radiation Protection (ICNIRP) [3] establishes the reference values for the exposure limits on human body. The main parameter is the Specific Absortion Rate (SAR) of energy, that measures the heating of human tissues. BTS exposure is extremely low for average mobile phone users, and SAR is almost exclusively due to MS emissions. Indeed, in that case, the SAR is proportional to the handset output power. Regulatory bodies must certify that MSs comply with ICNIRP limits. Based on ICNIRP recommendations, cell BTSs transmitting antennas should have exclusion zones ranging from a few centimeters for microcells to 10-20 meters for macrocells. Local administrations are in charge of controlling BTS installations. However, there is a growing number of publications reporting biological effects other than heating (see [4], [5]) that may occur at exposure levels below ICNIRP guidelines. These concerns often lead local regulatory bodies to even further constrain allowed BTSs locations. Nevertheless, if a BTS is far apart, MSs inevitably increase their emission power, and this issue has not been adequately addressed.

\section{THE OPNET MODELER}

Originally developed at the MIT (Massachusetts Institute of Technology), and introduced in 1987 as the first commercial network simulator, the OPNET Modeler (OPtimised Network Engineering Tool) is an environment for network modeling and simulation [6]. OPNET allows to design and study communication networks, devices, protocols, and applications.

The OPNET Modeler is organized as a hierarchical editor tool, resembling the actual structure of networks, equipments, and protocols. At the top of the hierarchy is the Project Editor, which is a graphical interface used to descibe the topology of a communication network. That is, the nodes and links among them. The Node Editor captures the architecture of 


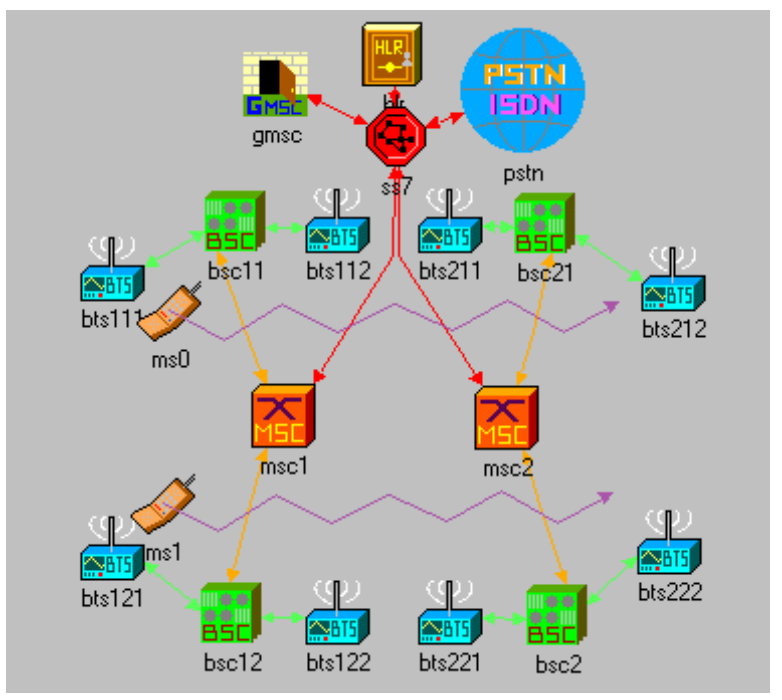

Fig. 1. OPNET's GSM model

these network components, depicting the data flow between functional elements (called modules). The modules are further detailed in the Process Editor. The Process Editor uses a powerful Finite State Machine (FSM) approach to support the specification, at any level of detail, of protocols, resources, applications, algorithms, and queuing policies. By means of the FSM the states and the event-driven transitions of the module elements are graphically defined. Each state of a process model contains $\mathrm{C} / \mathrm{C}++$ code, supported by an extensive library of functions designed for protocol implementation.

OPNET includes a wireless module [7], that extends the functionality of the core framework with high-fidelity modeling, simulation, and analysis of wireless networks. The wireless module implements many aspects of wireless transmissions, including:

- RF propagation (path loss with terrain diffraction, fading, and atmospheric and foliage attenuation).

- Interference.

- Transmitter/receiver characteristics.

- Node mobility, including handover.

- Interconnection with wire-line transport networks.

In addition, there exists a contributed GSM model for the OPNET suite [8]. It is quite detailed, focused in radio transmission aspects and signalling protocols. It implements the most important modules of a GSM network (see Figure 1). It also models the interconnection with the PSTN (Public Switching Telephone Network). Besides, the GSM model simulates the movement of mobile terminals across cells, and measures uplink and downlink received power levels. However, neither the appropriate propagation models, nor the GSM power control algorithm are implemented in this GSM model. We have introduced both of them in the implementation, to perform suitable simulations.

\section{PROPAGATION PREDICTION MODELS}

Urban and suburban environment propagation has been modelled by means of the Hata-Okumura model and the

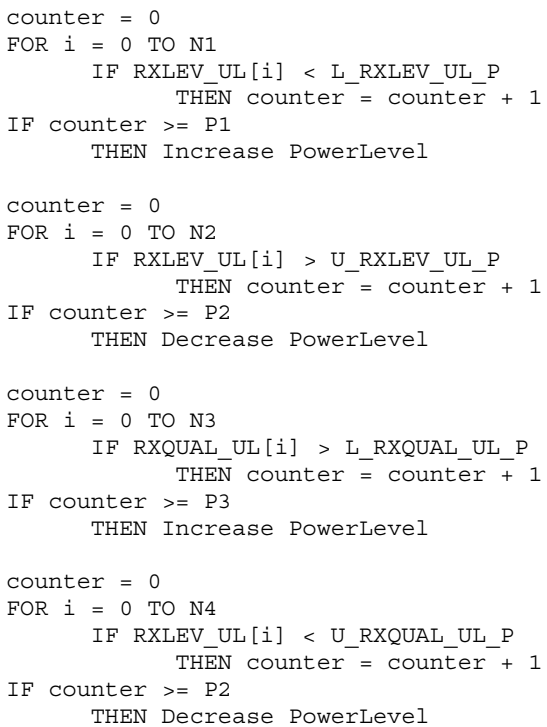

TABLE I

GSM POWER CONTROL Algorithm

Longley-Rice model, respectively.

Hata's model is one of the most widely used models for 900 $\mathrm{MHz}$ GSM signals prediction in urban areas [9]. This is an empirical formulation of the graphical losses data provided by Okumura, and it is valid from $150 \mathrm{MHz}$ to $1500 \mathrm{MHz}$. Hata presented the urban propagation loss as a standard formula and supplied correction equations for application to other situations. There are correction equations for small or medium sized cities and for large cities.

The Longley-Rice propagation prediction model (aka ITS, the Irregular Terrain Model) can be used in the frequency from $40 \mathrm{MHz}$ to $100 \mathrm{GHz}$, over different kinds of terrain in suburban areas [9]. One important modification to the Longley-Rice model deals with radio propagation in urban areas. This modification introduces an excess term, called the urban factor (UF), as an allowance for the additional attenuation due to urban clutter near the receiving antenna. So, this propagation model is suitable for $900 \mathrm{MHz}$ GSM in suburban installations.

The Hata propagation model is available as contributed model of the OPNET suite [8]. Besides, we have adapted the Longley-Rice propagation model implementation available at [10] for the OPNET engine.

\section{GSM POWER CONTROL ALGORITHM}

In this section we describe the GSM power control algorithm. The reference document is the GSM technical specification 05.08 - Annex A [11]. Power control mechanism objective is to control the transmission power level of the MS during calls, to fulfill the required uplink power level and quality, while keeping transmission power as small as possible. The GSM subsystems in charge of uplink power control are the BSC and the BTS, and its operation is as follows: 


\begin{tabular}{lll} 
PARAMETER & DESCRIPTION & $\begin{array}{l}\text { REFERENCE } \\
\text { VALUE }\end{array}$ \\
\hline L_RXLEV_UL_P & $\begin{array}{l}\text { RXLEV threshold on the uplink for power increase. Typical ranges from }-103 \\
\text { to }-73 \mathrm{dBm} .\end{array}$ & $-103 \mathrm{dBm}$ \\
\hline U_RXLEV_UL_P & RXLEV threshold on the uplink for power reduction. & $-90 \mathrm{dBm}$ \\
\hline L_RXQUAL_UL_P & RXQUAL threshold on the uplink for power increase. & $0 \%$ \\
\hline U_RXQUAL_UL_P & RXQUAL threshold on the uplink for power reduction. & $1.6 \%$ \\
\hline N1 & $\begin{array}{l}\text { The number of RXLEV_UL samples that the BSC shall store to compare with } \\
\text { the L_RXLEV_UL_P thereshold. Range [1, 31]. }\end{array}$ \\
\hline N2 & $\begin{array}{l}\text { The number of RXLEV_UL samples that the BSC shall store to compare with } \\
\text { the U_RXLEV_UL_P thereshold. }\end{array}$ \\
\hline N3 & $\begin{array}{l}\text { The number of RXQUAL_UL samples that the BSC shall store to compare with } \\
\text { the L_RXQUAL_UL_P thereshold. }\end{array}$ \\
\hline N4 & $\begin{array}{l}\text { The number of RXQUAL_UL samples that the BSC shall store to compare with } \\
\text { the U_RXQUAL_UL_P thereshold. }\end{array}$ \\
\hline P1 & Parameter used in threshold comparison process. \\
\hline P2 & Parameter used in threshold comparison process. \\
\hline P3 & Parameter used in threshold comparison process. \\
\hline P4 & Parameter used in threshold comparison process. \\
\hline
\end{tabular}

TABLE II

Thresholds AND PARAMETERS FOR THE GSM Power CONTROL Algorithm

Periodically, the BTS receives a SACCH burst ${ }^{1}$ from the MS (every $480 \mathrm{~ms}$ ). Upon reception, the BTS measures the received power and quality. The received power level is mapped to a integer value between 0 and 63, called RXLEV, and the received quality is mapped to a integer value between 0 and 7, called RXQUAL [11]. Besides, in the header of the received SACCH burst, the MS indicates its current power level [12].

The RXLEV and RXQUAL measured values, together with the MS power level are sent to the BSC in a message called Measurement Result. For every MS the BSC shall store at least the last 32 samples of the RXLEV and RXQUAL values. When a new Measurement Result message arrives (every $480 \mathrm{~ms}$ ), the BSC averages the value of the stored RXLEV and RXQUAL samples. Mean values are stored in the RXLEV_UL and RXQUAL_UL variables. Afterwards, the BSC performs the threshold comparison process. Table 1 summarizes this procedure, that is computed using the parameters and thresholds shown in Table 2. The threshold comparision algorithm decides if MS power must be increased or decreased. Nevertheless, the GSM standard does not specify how to compute how many power steps $(1$ power step $=2$ $\mathrm{dB}$ ) the power must be corrected. In the simulations we have choosed to increment or decrement the power by one step, i.e. $2 \mathrm{~dB}$. When the new power value is calculated, the BSC transmits it to BTS in a MS Power Control message. Finally, the BTS sends the new power level to the MS in the header of the next SACCH burst.

This procedure has been implemented and integrated into the OPNET GSM model.

\section{Simulation RESUlts}

In this section we describe the simulations performed and the results achieved. Figure 2 shows the experiments set up. Two scenarios were considered. Namely,

\footnotetext{
${ }^{1}$ The Slow Associated Control Channel (SACCH) bursts are multiplexed with traffic or dedicated control channel bursts, and are used for channel maintenance porpuoses, e.g. power control signaling.
}

1) Urban scenario. The BTS is placed in the urban area (Fig. 2.(a)). MS trajectory goes from $X=-2700 \mathrm{~m}$. to $X=+2700 \mathrm{~m}$. at a speed $1 \mathrm{~m} / \mathrm{s}$. In this scenario we used the Hata's propagation model for small cities, both in the uplink and in the downlink.

2) Suburban scenario. In this case, the BTS is placed in the suburban area (Fig 2.(b)), $5 \mathrm{~km}$ away from the urban area. The MS path starts in $\mathrm{X}=5000 \mathrm{~m}$., and ends in $X=10400 \mathrm{~m}$., the travel speed is $1 \mathrm{~m} / \mathrm{s}$. In this scenario we used the Longley-Rice propagation model, both in the uplink and in the downlink. Urban terrain has been taken into account by adding the urban factor, to further increase the pathloss due to urban clutter near the receiving antenna [13].

In all the tests we set the power class of the MS to 4 (i.e. the nominal maximum output power is $2 \mathrm{~W}(33 \mathrm{dBm}))$, and the power class of the BTS to 5 (i.e. the nominal maximum output power is $20 \mathrm{~W}$ ). Additonally, the power control algorithm uses the reference values shown in Table 2 (parameters P1P4 and N1-N4 have been extracted from the GSM technical specification 05.08 - Annex A [11]).

Figures 3 shows the MS transmission power (i.e. the uplink power) versus the mobile position, for the urban and suburban scenarios, respectively. In addition, the average uplink power in the urban scenario was $25.6333 \mathrm{dBm}(365.8726 \mathrm{~mW})$, and in the suburban scenario was $33 \mathrm{dBm}(2000 \mathrm{~mW})$. Note that in the latter scenario the MS always transmitted at the maximum power. This is because the distance between the MS and the BTS was so large that the GSM power control algorithm led the MS to transmit always at the maximum power to fulfill the required uplink quality. However, in the urban scenario the distance between the MS and the BTS was lower, and consequently the MS uplink power was variable.

In the future we want to conduct additional experiments using different mobility profiles for the MS, different urban cells sizes, and changing the distance from BTS to urban area, in suburban scenarios. 


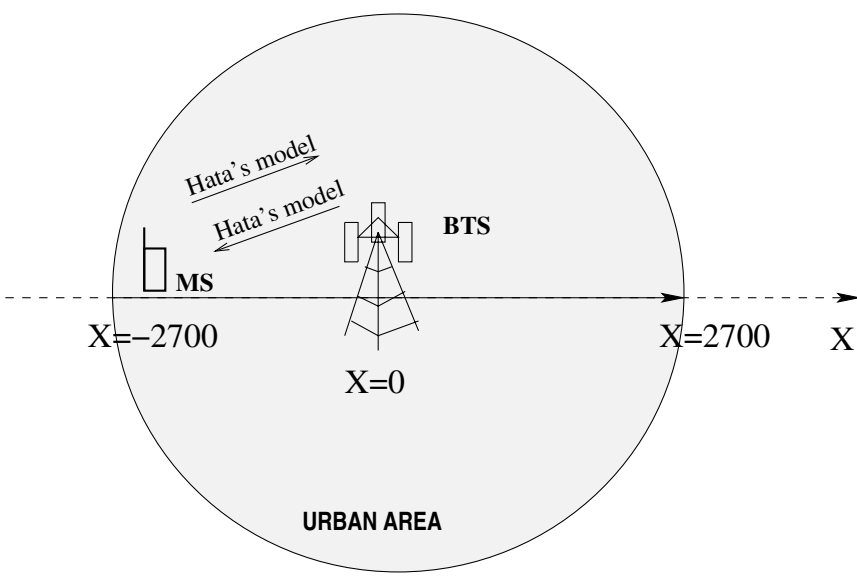

(a) Urban scenario

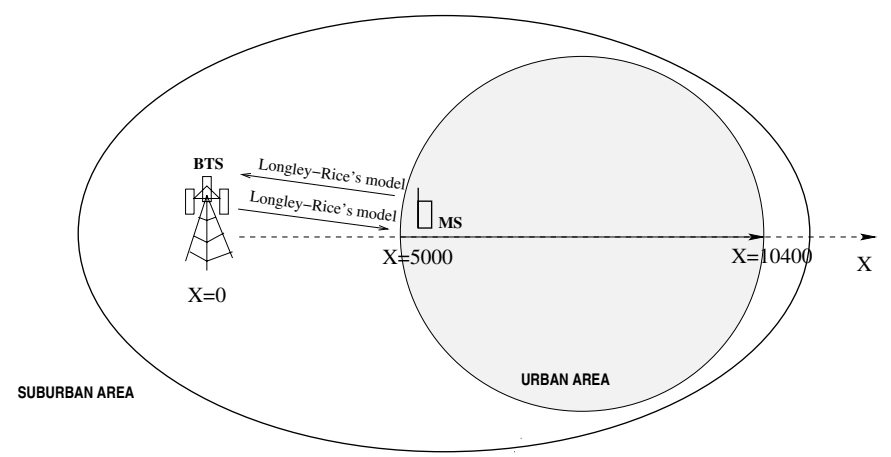

(b) Suburban scenario

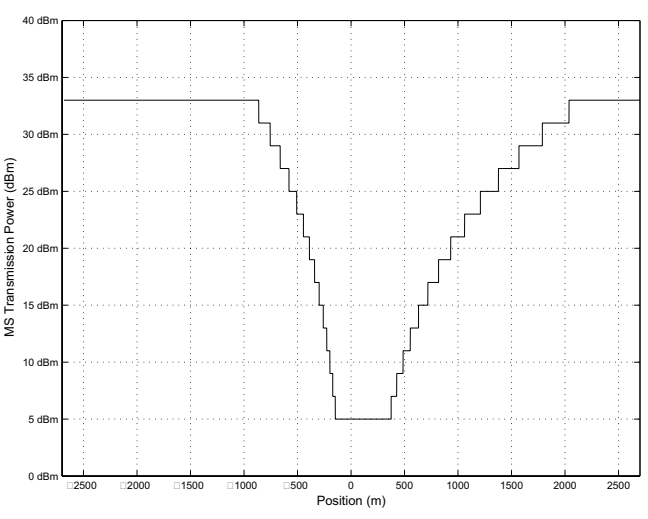

(a) Urban scenario. Tx. Power

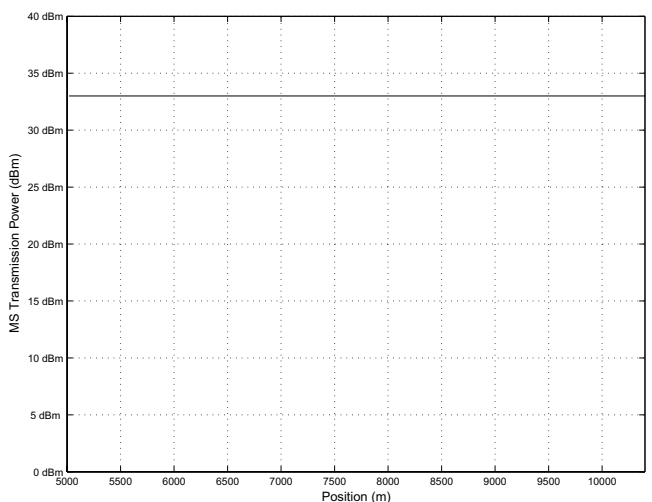

(b) Suburban scenario. Tx. Power
Fig. 2. Scenarios of simulation

\section{CONCLUSIONS}

In short, simulations show that the transmitted power when the BS is located in the suburban area is $7.277 \mathrm{~dB}$ greater than a standard BTS installation. That is, users are exposed to $446 \%$ more power (1634.12 $\mathrm{mW} / 365.8726 \mathrm{~mW}$ ) than of a standard BTS installation. This notable increment in the exposure level may raise human health safety concerns. Therefore, relocating antennas of cellular systems out of the urban environment has a worse effect than the one that they are trying to alleviate. Alternatives to the conventional GSM architecture must be designed in order to allow BS to be installed outside populated areas, while keeping a relative small MS transmission power. With a suitable solution we could reduce human exposure to GSM radiation levels.

\section{ACKNOWLEDGMENTS}

This work has been funded by the Spanish Economy, Industry and Innovation Council under the SOLIDMOVIL project (2I04SU044), by Fundación Seneca (Región de Murcia, Spain) ARENA project (00546/PI/04) and by the Spanish Research Council under the ARPaq project (TEC2004-05622C04-02/TCM). The authors are also indebted to OPNET Technologies Inc. for granting licenses under the OPNET University program.

\section{REFERENCES}

[1] Moulder, J. Cellular Phone Antennas (Mobile Phone Base Stations) and Human Health web site. [Online]. Available: http://www.mcw.edu/gcrc/cop/cell-phone-health-FAQ/toc.html

[2] United Nations International EMF Project. [Online]. Available: http://www.who.int/peh-emf/project/en/

[3] The International Commission on Non-Ioninzing Radiation Protection. ICNIRP. [Online]. Available: http://www.icnirp.de/

[4] Salford, G. , Brun, A., Eberhardt, J., Malmgren, L., Persson, B. (2003). Nerve Cell Damage in Mammalian Brain after Exposure to Microwaves from GSM Mobile Phones. Environmental Health Perspectives, 11:7.

[5] Reuters. (2004, Dec.). Study: Cell phones scramble DNA. [Online]. Available: http://dynamics.org/Altenberg/MED/CELL_PHONES/21021039_3-5498198.html

[6] OPNET Modeler. Available: http://www.opnet.com/products/modeler/home.htm

[7] OPNET Wireless Module. Available: http://www.opnet.com/products/modules/wireless_module.htm

[8] OPNET contributed models. Available: http://www.opnet.com/support/cont_models.htm

[9] Rappaport, T. S. (2002). Wireless Communications, Principles and Practice, second edition. Prentice Hall.

[10] Irregual Terraim Model (ITM) (Longley-Rice) Available: http://flattop.its.bldrdoc.gov/itm.html

[11] 3GPP (TS 05.08) Digital cellular telecommunications system (Phase 2+); Radio subsystem link control 3GPP Technical Specification 05.08 v. 8.22 .0

[12] 3GPP (TS 04.04) Digital cellular telecommunications system (Phase 2+); Layer 1 - General Requirements 3GPP Technical Specification 04.04 v. 8.1.2

[13] Longley, A. G. (1978). Radio Propagation in Urban Areas. OT Report 78-144. United States Department of Commerce, Office of Telecommunications. 